\title{
Atomistic mechanisms of binary alloy surface segregation from nanoseconds to seconds using accelerated dynamics
}

\author{
Richard B. Garza ${ }^{1,2}$, Jiyoung Lee ${ }^{3,4}$, Mai H. Nguyen ${ }^{3}$, Andrew Garmon ${ }^{5,6}$, Danny \\ Perez ${ }^{5}$, Meng Li ${ }^{2}$, Judith C. Yang ${ }^{2}$, Graeme Henkelman ${ }^{3,4}$, Wissam A. Saidi ${ }^{*}$ \\ ${ }^{1}$ Department of Mechanical Engineering and Materials Science, University of \\ Pittsburgh, Pittsburgh, PA \\ ${ }^{2}$ Department of Chemical and Petroleum Engineering, University of Pittsburgh, \\ Pittsburgh, PA \\ ${ }^{3}$ Department of Chemistry, University of Texas at Austin, Austin, TX \\ ${ }^{4}$ Oden Institute for Computational Engineering \& Sciences, University of Texas at \\ Austin, Austin, TX \\ ${ }^{5}$ Theoretical Division T-1, Los Alamos National Laboratory, Los Alamos, NM \\ ${ }^{6}$ Department of Physics \& Astronomy, Clemson University, Clemson SC \\ * Corresponding author. E-mail: alsaidi@pitt.edu
}

\begin{abstract}
Although the equilibrium composition of many alloy surfaces is well understood, the rate of transient surface segregation during annealing is not known, despite its crucial effect on alloy corrosion and catalytic reactions occurring on overlapping timescales. In this work, $\mathrm{CuNi}$ bimetallic alloys representing (100) surface facets are annealed in vacuum using atomistic simulations to observe the effect of vacancy diffusion on surface separation. We employ multitimescale methods to sample the early transient, intermediate, and equilibrium states of slab surfaces during the separation process, including standard MD as well as three methods to perform atomistic, long-time dynamics: parallel trajectory splicing (ParSplice), adaptive kinetic Monte Carlo (AKMC), and kinetic Monte Carlo (KMC). From nanosecond (ns) to second timescales, our multiscale computational methodology can observe rare stochastic events not typically seen with standard MD, closing the gap between computational and experimental timescales for surface segregation. Rapid diffusion of a vacancy to the slab is resolved by all four
\end{abstract}


methods in tens of ns. Stochastic re-entry of vacancies into the subsurface, however, is only seen on the microsecond timescale in the two KMC methods. Kinetic vacancy trapping on the surface and its effect on the segregation rate are discussed. The equilibrium composition profile of $\mathrm{CuNi}$ after segregation during annealing is estimated to occur on a timescale of seconds as determined by $\mathrm{KMC}$, a result directly comparable to nanoscale experiments.

\section{Introduction}

Alloy surfaces are typically enriched with one of their constituent elements, particularly in the top layers, due to differences in the surface energies of the pure metals. This surface segregation process leads to metallic de-mixing, which is of relevance to many different fields of research such as catalysis and metallurgy, considering that in situ transformations can affect the chemical activity or structural integrity gained from homogeneously alloying pure metals together. Alloy surface segregation and ordering have been measured experimentally both in vacuum and gas environments, with differences in the equilibrium alloy composition induced near the exposed top layers for many bimetallic alloys[1-6]. Consequently, studies of alloy surface transformations are influenced by prior phase separation in vacuum during pre-treatment. A decoupling of the experimental environment from the pre-treatment environment is required to understand the transient effect on non-equilibrium surface composition, since nanoscale elemental mapping is not feasible on relatively short microsecond $(\mu \mathrm{s})$ timescales even using electron and X-ray diffraction.

Molecular dynamics (MD) simulations can generally resolve atomic transitions on the picosecond (ps) to nanosecond (ns) timescales. While these short timescale dynamics can be easily investigated using energetic models based on either classical force fields or first-principles 
methods, longer timescales that are often more relevant experimentally are challenging to realize with conventional resources and techniques[7-9]. As shown in the current work, alloy surface segregation occurs over millisecond (ms) timescales that are impractical to obtain using conventional MD simulations. This goal can be achieved with accelerated methods including adaptive kinetic Monte Carlo (AKMC) and temperature-accelerated dynamics (TAD)[10-12]. These two methods were employed in a multiscale approach to study surface segregation in ceramics (rocksalt oxides) during oxidation[13]. Also, more recently, surface segregation and timescales in PdAu nanoparticles have been studied using AKMC[14]. However, alloy segregation and timescales related to the metallic dopant dynamics are not well understood in nanoscale thin films, as these require larger model systems to accurately simulate the region between bulk and exposed surface. A recent KMC study investigated the dynamics in bulk NiFe solid solution, reporting that the vacancy migration barrier is highly influenced by the local composition, behavior that the authors predict will influence the rate of microscale phase transformations[15]. Previously, only the dependence of activation energy on overall alloy composition could be predicted [16]. The failure of these global models for concentrated solid solutions is known, though they may be corrected by sampling transition energies in differing chemical environments[17].

In this study, we model the non-equilibrium surface segregation process by employing multiscale simulations methods that probe the dynamics from ps to second timescales. We focus our investigations on cupronickel $(\mathrm{CuNi})$, which exhibits surface segregation at elevated temperatures in vacuum, enriching with $\mathrm{Cu}$ near the surface and $\mathrm{Ni}$ in the bulk[18, 19]. The CuNi alloy is of interest for different applications with extreme environmental conditions 
including marine settings due to its resistance to corrosion by seawater[20, 21], as well as a hightemperature catalyst for thermal $\mathrm{CO}_{2}$-to-syngas-to-fuels conversion[22-25].

To date, the transient dynamics of segregation in $\mathrm{CuNi}$ have not been investigated. Previous studies have employed Monte Carlo (MC) to show that the Ni solute concentration is significantly decreased in the top three surface monolayers for all slab orientations, approaching its value in the bulk at a depth of four or five atomic layers below the surface[26-28]. While MC simulations describe the systems in equilibrium, they do not give a timescale for the segregation process. In contrast, MD can provide a timescale for dynamics, but it has not been utilized before to study transient dynamics of the segregation process. Limited investigations of dislocation slip under applied stress (work hardening) or melting in $\mathrm{CuNi}[29,30]$ have been carried out using MD because these processes occur over ns timescales, yet the impact on larger scale reordering on experimental timescales was not explored in those studies. Thus, the mechanism, as well as the timescale, for transformation from a randomly mixed to ordered alloy on the nano- and microscales remains unclear.

Accelerated methods can provide insight on longer timescales into atomistic mechanisms of surface segregation phenomena. Previously, AKMC investigations of the segregation kinetics of PdAu nanoparticles showed greater kinetic stability due to reduced strain in the mixedphase[14]. In the present work, we probe segregation dynamics in planar, (100) CuNi surfaces, employing conventional MD and three accelerated dynamics methods: parallel trajectory splicing (ParSplice), AKMC, and KMC with kinetic barriers derived from a cluster expansion. ParSplice affords accurate system evolution up to $\sim 10 \mu$ s, while $\mathrm{AKMC}$ and $\mathrm{KMC}$ simulate longer timescales up to ms and seconds, respectively. 
ParSplice [11] extends MD simulation times by leveraging parallel computers to carry out parallelization in the time domain, in contrast to the usual domain decomposition approaches that operate in the space domain $[31,32]$. Thus, with ParSplice it is possible to simulate small systems over very long timescales, again in contrast to conventional parallelization approaches that are efficient at spatially decomposing large systems simulated over short timescales. This is accomplished by concurrently generating a large number of independent, short trajectory segments using a procedure that guarantees these segments can be assembled into a longer stateto-state trajectory that is statistically correct[33]. Periodic quenching is used to identify transitions between different metastable states and segment terminations. It can be shown that ParSplice trajectories can become arbitrarily accurate by adjusting the estimate of the so-called correlation time of the dynamics, at the expense of a computational overhead [34]. When the dynamics follow from a sequence of rare events, ParSplice can provide a computational speedup that scales with the number of processors used; it is therefore especially powerful when deployed on massively parallel computers.

KMC-based methods do not have a fixed timestep; instead, they find the time elapsed for the first escape from one state to another, allowing for large periods of vibrational motion in the atomic system to be bypassed[14, 35]. These escape times correspond to reaction rates, which are calculated adaptively or "on the fly" to construct an AKMC state model: nothing in the output event table is predefined or assumed from prior knowledge[12, 14, 35]. AKMC uses minimum-mode following searches or high-temperature MD to construct this event table: the transition state energies (activation barrier heights) are found using single-ended saddle point finding algorithms such as the dimer method[36-38]. To achieve further acceleration while maintaining as much of this accuracy as possible, in the present study, we use off-lattice 
dynamics from AKMC to fit a more approximate, lattice-based KMC model, which functionally depends on the Ni-coordination via a cluster expansion.

Our multiscale approach produces a hierarchy of trajectory data for the segregation process over a broad range of timescales. To represent trajectory data on varying scales and fidelities, we track physical properties including the local defect chemical environment and its influence on the correlated rates of segregation and vacancy migration. Further, we find that segregation in the top layer is a function of the rare re-entry of a vacancy from the surface to the subsurface, which only occurs with a frequency of $10 \mu$ s due to a high kinetic barrier, greatly increasing the time required for the surface to be completely depleted of $\mathrm{Ni}$. We also determine that the number of dopant atoms in the $1^{\text {st }}$ and $2^{\text {nd }}$ coordination shells around the point defect alters its migration energy, affecting the rate of composition change. The consistency of system evolution during segregation by thermal annealing across all the accelerated methods is examined on many timescales; this evidence supports each technique's further use in multiscale simulations in combination with the data processing methods used in this work.

\section{Methodology}

\subsection{Validation of the Embedded-Atom Method Potential}

A reliable interatomic potential is required to obtain accurate MD of surface segregation and equilibrated structures. Here, we employed the embedded atom model (EAM) potential of Fischer and collaborators, which is designed to model $\mathrm{CuNi}$ phase segregation across grain boundaries and is parameterized with surface energies, lattice constants, vacancy migration energies, and relevant quantities governing the rate of metallic phase separation [39]. We first verified the applicability of this potential to study CuNi surface segregation by comparison of 
calculated surface energies with those obtained from spin-polarized DFT calculations carried out using VASP with the Perdew-Burke-Enzerhof (PBE) exchange-correlation functional[40-44], see SI Appendix A, Table A1. Further, we verified that the segregation of Ni to the bulk-from the $1^{\text {st }}$ to the $2^{\text {nd }}$ or $3^{\text {rd }}$ layer - is always favorable i.e. $\Delta \mathrm{E}_{\mathrm{Ni} \text {,surf } \rightarrow \text { interior }} \approx-0.4$ to $-0.3 \mathrm{eV}$ from DFT and EAM, also in agreement with previous ab initio calculations [45]. Further, we found good agreement with the previously reported anisotropic, thermodynamic tendency for $\mathrm{Ni}$ to segregate to exposed $<100>$ and $<110>$ facets in favor of $<111>$ surfaces $[19,27]$.

\subsection{Slab Models and Initial State for Annealing}

We employed slab models of the (100) surface termination that are composed of 216 and 384 atoms by randomly substituting $\mathrm{Cu}$ with $\mathrm{Ni}$. These slabs were 12 monolayers in thickness with $3 \times 3$ and $4 \times 4$ surface periodicity; nearly doubling the vacancy concentration (1:216 vs. 1:384 atoms) did not affect our observed mechanisms or energetics. Thus, systems of smaller surface periodicity $(3 \times 3,216$ atoms) were simulated to realize the longest timescales for higher fidelity time-averaging and activation energy histograms. $\mathrm{MC}$ simulations across the composition range of $2.7-16 \mathrm{at} \% \mathrm{Ni}$, and temperature range of $300-700 \mathrm{~K}$ showed no detectable variation in the amount of Ni segregated. For this reason, we chose to exclusively simulate systems with 16 at $\%$ $\mathrm{Ni}$ at $500 \mathrm{~K}$ with the accelerated MD methods.

The equilibrium composition profile of the CuNi (100) surface alloy is determined using MC. The method attempts $10^{6} \mathrm{MC}$ swaps of $\mathrm{Cu}$ and $\mathrm{Ni}$ atoms with subsequent minimization and an acceptance probability as determined via the Metropolis algorithm[46]. MC composition profiles are found to be consistent with similar reported profiles [18, 26-29, 47] across a range of compositions and temperatures, see SI Appendix B. Such agreement with previous 
computational and experimental results is a further validation of the reasonable accuracy of the EAM potential.

\subsection{Multiscale Simulation Hierarchy}

The methods employed in our multiscale hierarchy are described as follows, in order of accessible timescale: molecular dynamics in the NVT ensemble was generated using a Langevin thermostat, as implemented in the LAMMPS code[48]. Many instances of LAMMPS were then orchestrated by the EXAALT/ParSplice code [http://gitlab.com/exaalt] to carry out long-time ParSplice simulations. Direct MD realized ns of simulated time using the velocity Verlet algorithm and modest resources (4 processors). ParSplice accelerated these dynamics up to $\mu \mathrm{s}$ timescales using 224 processors. Both standard MD and ParSplice were carried out in the same way: after initializing particle velocities according to a Boltzmann distribution representing the target temperature, the systems were equilibrated with a 2 fs timestep and a 1 ps temperature relaxation time for $200 \mathrm{ps}$. After the equilibration phase of $\mathrm{MD}$, a further $400 \mathrm{~ns}$ were simulated to constitute the production phase, while ParSplice accelerated this simulation time up to $35 \mu \mathrm{s}$. Minimization was done with the conjugate gradient algorithm using convergence criteria of $10^{-8}$ $\mathrm{eV}$ and $10^{-6} \mathrm{eV} / \AA$ for the energy and forces[49], respectively. Quenching is necessary for ParSplice to identify state transitions and to properly terminate each parallel MD replica.

In contrast to ParSplice and MD, the AKMC and KMC models use a non-constant timestep, which varies to match the timescale of the first escape time from a given state. Over longer timescales, up to many seconds, $\mathrm{KMC}$-based acceleration approaches the segregation found with $\mathrm{MC}[12,35]$. KMC methods simulate the time evolution of the system, requiring a predetermined event table in which the kinetic rate of each event is approximated through the 
Arrhenius relation to the pre-calculated activation energy. At each step, a random event $i$ is selected from the table in the order from 1 to $i$ with the condition

$$
\sum_{1}^{i-1} r_{i-1}<p_{1} \sum_{1}^{N} r_{N} \leq \sum_{1}^{i} r_{i}
$$

where $\sum_{1}^{i} r_{i}$ is the sum of the rate from event 1 to event $i, \sum_{1}^{i-1} r_{i-1}$ is the sum of the rate from event 1 to event $i-1, p_{1}$ is a random number between 0 and 1 , and $\sum_{1}^{N} r_{N}$ is the total rate of the event table. Time is then incremented by

$$
\frac{-\ln \left(p_{2}\right)}{\sum_{1}^{N} r_{N}}
$$

where a random number $p_{2}$ is drawn between 0 and 1 . AKMC allows the system to find all potential events without a need of a predetermined event table [45]. In order to search for events, AKMC uses high-temperature MD and the climbing-image nudged elastic band (CI-NEB) approach to calculate the saddles for the new states using the EON software[50].

To reach even longer timescales with $\mathrm{KMC}$, an energy estimation based on the local environment of the vacancy was generated by a cluster expansion. The energy was predicted to be dependent on the concentration of $\mathrm{Ni}$ and $\mathrm{Cu}$ located in the first nearest neighbor shell of the vacancy. In the FCC CuNi alloy, there are eight nearest neighbors for the vacancy on the surface and twelve nearest neighbors for the vacancy in the subsurface. Sequentially, the energy-fitting model was used to determine the barrier and rate of the events in the event table based upon the trajectory data from our AKMC simulations. More information about the cluster expansion method is provided in the SI, Appendix C. Further, we found satisfactory agreement between $\mathrm{AKMC}$ and $\mathrm{KMC}$ by carrying out the dynamics up to $300 \mu \mathrm{s}$, further validating the KMC model (see SI, Appendix D). 
Finally, the concept of an equilibrium rate was introduced to further accelerate the timescale accessible by KMC. In this approximation, no rate was allowed to be larger than the specified equilibrium rate with the assumption that all states connected by rates faster than the equilibrium rate should already be in equilibrium. In the case of $\mathrm{CuNi}$ segregation, the planar diffusion of a vacancy on the exposed surface is rapid, equilibrating on much shorter timescales than for defect re-entry to the subsurface to occur even once. Ultimately, the effect of an artificial equilibrium rate in $\mathrm{KMC}$ simulations is that these key transitions can be sampled more effectively instead of the many horizontal transitions that do not alter the composition with respect to surface depth.

In both $\mathrm{AKMC}$ and $\mathrm{KMC}$ simulations, the temperature was set to $500 \mathrm{~K}$ and the prefactor for the rates was fixed at $5 \times 10^{12} \mathrm{~s}^{-1}$. The optimizer used in AKMC was L-BFGS, with a convergence criterion of $0.01 \mathrm{eV} / \AA ̊[49]$. System evolution from AKMC reached ms timescales running for a week on 24 cores. The following KMC timings are the average of five separate runs in both cases: KMC realized seconds of simulation time running on a single processor for 2 hours with the added equilibration rate. $\mathrm{KMC}$ without the equilibrium rate was only simulated up to $10 \mathrm{~ms}$, since only one minute of wall clock time was necessary before the surface vacancy trapping described above halted Ni segregation in the system.

\section{Results and Discussion}

The equilibrium composition profiles obtained with MC (Figure 1) exhibit Ni migration out of the top three surface layers, while the concentration of the fourth and fifth layers approach the bulk value. This trend did not quantifiably vary with changes in system temperature or Ni concentration. The near-surface Ni concentration observed with the EAM potential agrees with the profiles derived from previous MC simulations [18, 26-29] as well as experiments[1-3, 19]. 


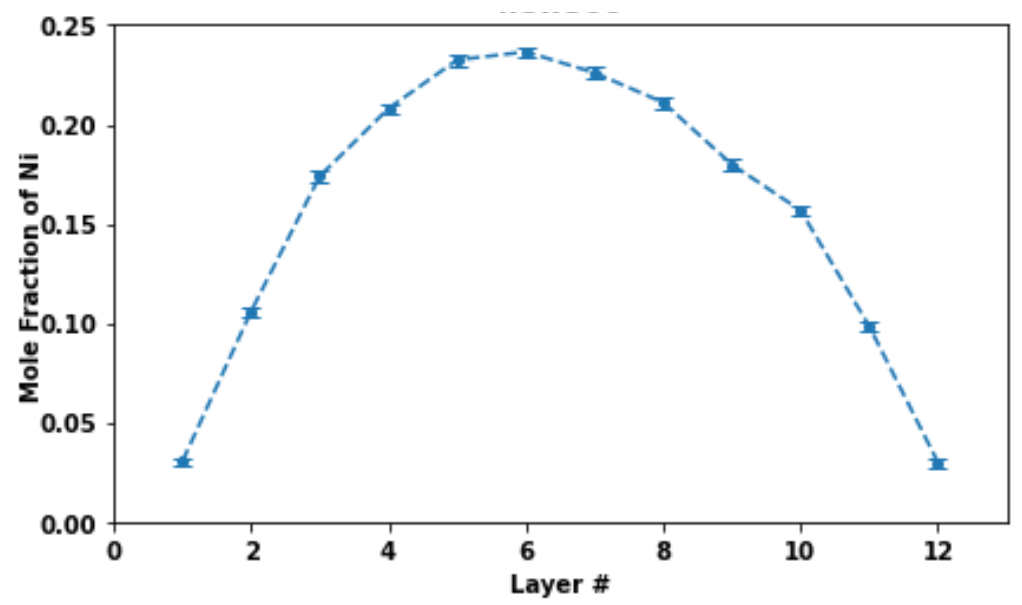

Figure 1: Ni composition profiles of $\mathrm{Cu}-16 \mathrm{at} \% \mathrm{Ni}(100)$ surface as a function of layer depth after MC annealing at $500 \mathrm{~K}$. The slab has 12 layers with layers 1 and 12 exposed to the vacuum. Composition is normalized by the number of atoms in a pristine $\mathrm{FCC}<100>$ layer (18) resulting in a mismatch between the bulk composition of layer $6(\sim 22 \mathrm{at} \% \mathrm{Ni})$ and the overall composition (16at\% Ni).

Next, we probed the segregation dynamics using MD, ParSplice, AKMC, and KMC starting from the same initial configuration of the random alloy. Lattice vacancies are the primary defect responsible for alloy segregation: the only other mechanism, self-interstitial migration of metal atoms to octahedral or tetrahedral sites, is destabilized by greater formation and migration energies than those for the point defect (vacancy)[51]. Hence, our simulations included a single vacancy to facilitate surface segregation. 

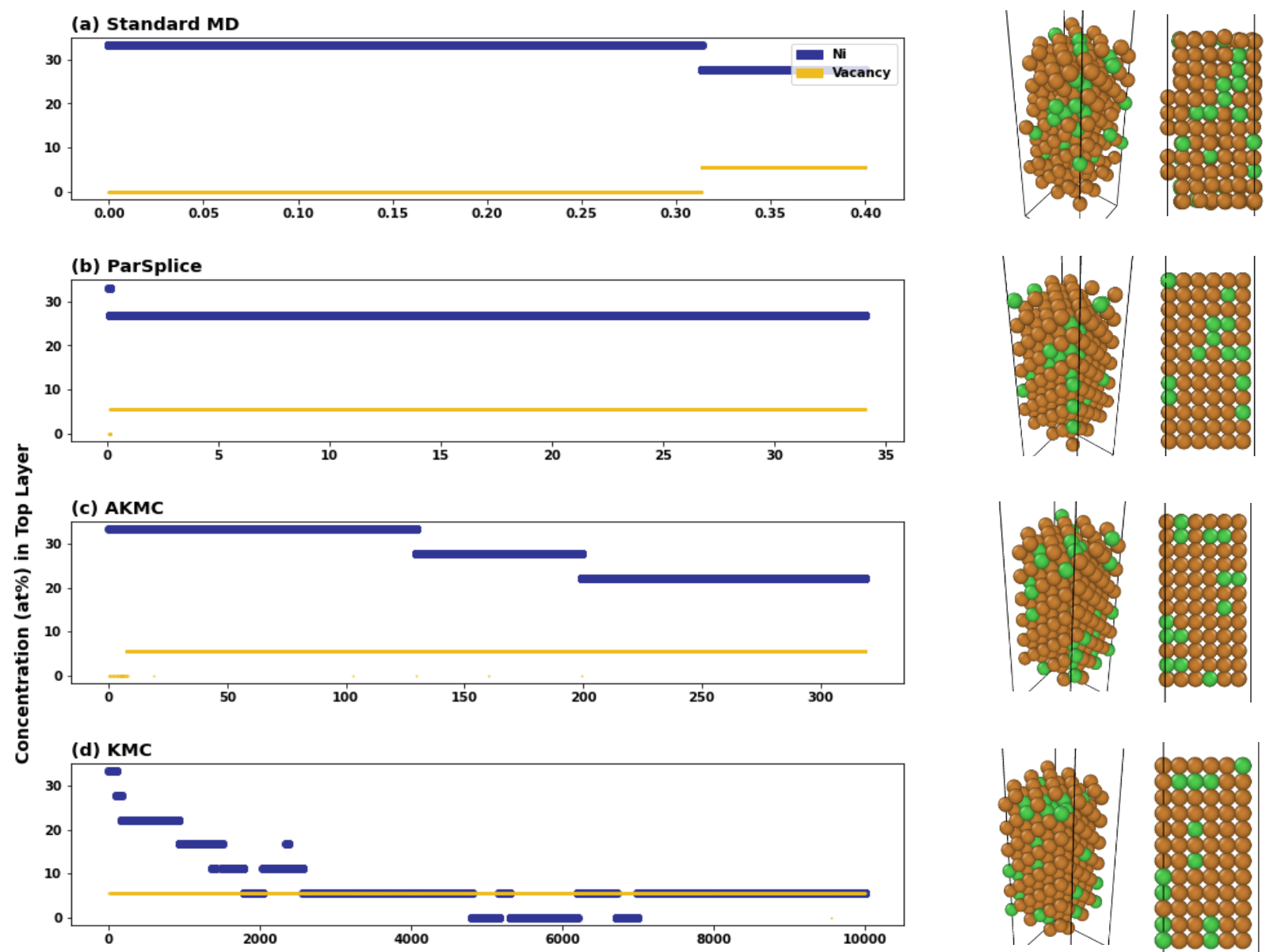

(e) $\mathrm{KMC}$ (eq. rate $=\mathbf{4 0} / \mu \mathrm{s})$
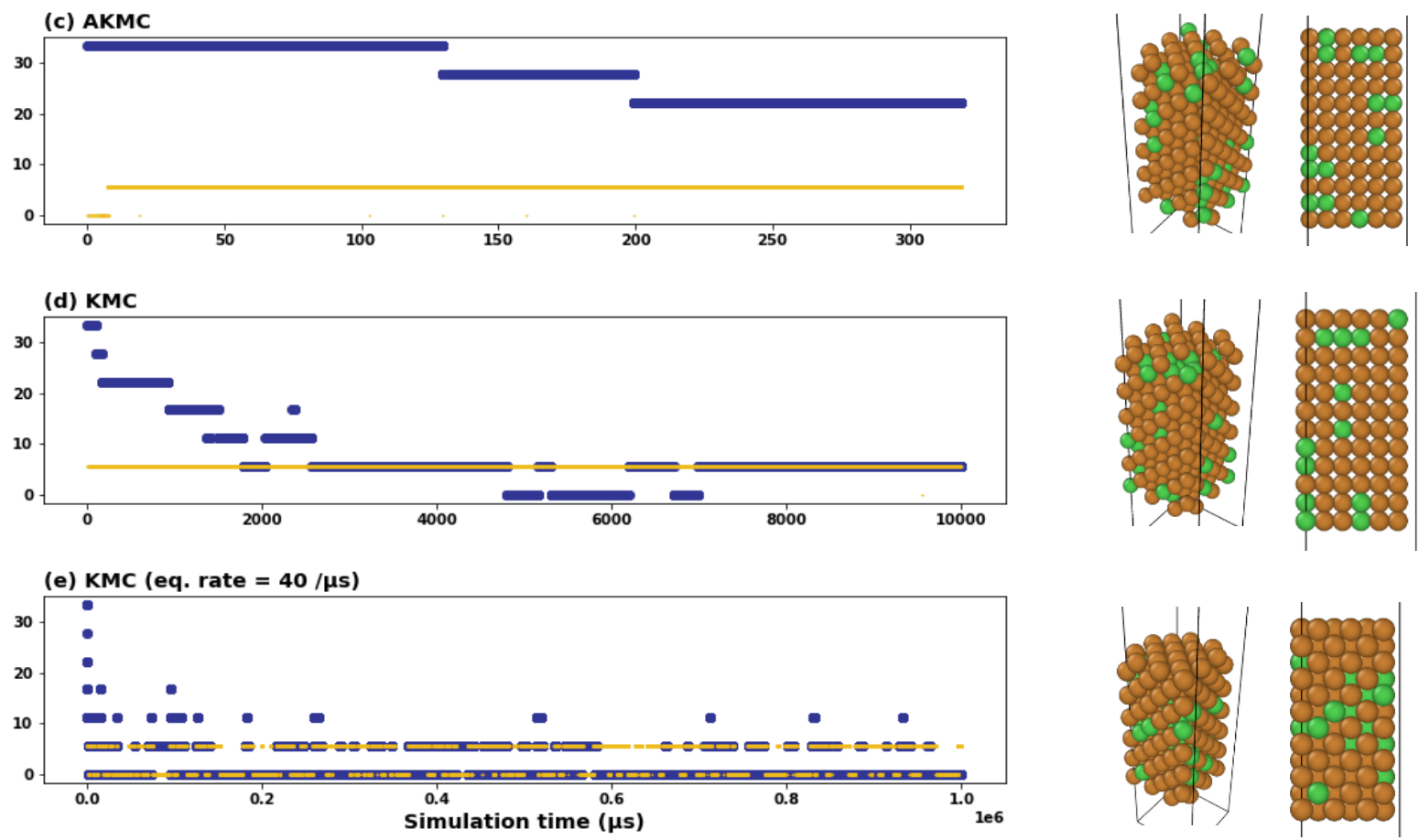

Figure 2: (left) $\mathrm{Ni}$ content in the top layer of $\mathrm{Cu}-16 \mathrm{at} \% \mathrm{Ni}(100)$ with a vacancy for (a) standard MD, (b) ParSplice, (c) AKMC, (d) KMC, and (e) KMC with applied equilibrium rate.

Composition is normalized as in Figure 1. (right) Orthographic and side views of the final slab model from each simulation are provided for reach simulation.

Figure 2 shows the composition over time within the top layer of our $\mathrm{Cu}-16 \mathrm{at} \% \mathrm{Ni}(100)$ slab model for a single simulation of each type within our methodology. The concentration of $\mathrm{Ni}$ 
atoms in the top surface layer decreases over time as it transitions from a uniform distribution to that of equilibrium, as shown by the MC simulations in Figure 1. The accessible simulation time increases sequentially for each method (MD, ParSplice, AKMC and KMC). In MD (Figure 2a), the vacancy diffuses to the surface at $0.3 \mu$ s, displacing a $\mathrm{Ni}$ atom to the subsurface; up to the total time of $0.4 \mu$ s the vacancy remains trapped on the surface so that the Ni concentration does not change. With over thirty different MD simulations, we observed that the time for vacancy percolation to the surface was consistently less than $1 \mu \mathrm{s}$, resulting in similar degrees of segregation as shown in Figure 2a.

ParSplice (Figure 2b) shows similar dynamics as in standard MD, with vacancy migration to the surface in a fraction of a $\mu$ s. Throughout the simulation, the ParSplice trajectory visited 8,278 topologically unique states while making 37,458 transitions, the vast majority of which occurred after the vacancy had reached the surface. The parallel acceleration of ParSplice increased the timescale from the standard MD simulation by a factor of $\sim 100$ up to $35 \mu \mathrm{s}$, but even at this longer timescale the vacancy remained trapped on the surface and no additional $\mathrm{Ni}$ segregation was observed. As expected, ParSplice is consistent with MD on timescales where they overlap $(0.4 \mu \mathrm{s})$ not only in terms of bulk vacancy diffusion but also the timescale at which the vacancy diffuses to the surface. We have repeated the ParSplice simulations dozens of times with different random seeds observing early vacancy diffusion to the surface (before $1 \mu \mathrm{s}$ ) in nearly all trials, just as with MD.

With AKMC, we increased the simulation timescale by another order of magnitude (Figure 2c): a total of 144,597 transitions evolved the system through 32,369 unique states (a similar ratio of transitions to new states found as in ParSplice). Note that our AKMC approach uses coarse-graining following the MC with absorbing Markov chains (MCACM) method, 
allowing many more transitions to be considered via an analytic solution to the rate equations[52]. From the AKMC dynamics, we can observe events in which the vacancy moves from the surface to the subsurface at a timescale of roughly $50 \mu$ s. Over the simulation time of $300 \mu$ s, five such events were observed resulting in two $\mathrm{Ni}$ atoms and $3 \mathrm{Cu}$ atoms migrating from the surface to the subsurface.

With our KMC model (Figure 2d), we executed 4.7 million transitions generating $10 \mathrm{~ms}$ of simulated time. Over these timescales, the system appears to approach equilibrium, with fluctuations in the surface concentration between 0 and $5 \% \mathrm{Ni}$. However, what is not obvious from these plots is that the vacancy spends all of its time in the first and second layer, so that $\mathrm{Ni}$ segregation only occurs between the surface and subsurface layers. This behavior originates from a disparity in barrier heights that embodies the "low-barrier problem", more aptly referred to as the "heterogeneous barrier" problem. Dynamics with a mix of low and high activation barriers are inherently more difficult to accelerate since groups of states interconnected by low barriers will always dominate the trajectory during naïve state space exploration[ $[8,11]$. The barrier for vacancy surface diffusion is $0.4-0.5 \mathrm{eV}$, whereas the barrier for the vacancy to go subsurface is 0.8-0.9 eV. Thus, we are simulating on the order of a million KMC steps with the vacancy mostly diffusing on the surface for one subsurface diffusion event, offering a low chance for segregation to occur. Even factoring in the small cost of each KMC step, this makes it impossible to simulate an equilibrium distribution of $\mathrm{Ni}$ in the top three layers.

To further accelerate the dynamics and mitigate the "heterogeneous barrier" problem, in our final simulation (Figure 2e), we perform KMC with the equilibrium rate approximation described in the methods section, realizing 160 million transitions to reach a simulated time of 1 s. Here, the idea is that the vacancy will quickly reach local equilibrium diffusing in the top 
layer, and no new states of interest are explored until subsurface diffusion occurs. Since vacancy surface diffusion occurs on a timescale of ns, and diffusion of the vacancy to the subsurface occurs on a timescale of $\mu \mathrm{s}$, we chose an equilibrium rate of $40 / \mu$ s to slow the surface diffusion and accelerate the diffusion of the vacancy to the subsurface and below. Figure 2e shows that on a timescale of seconds, the surface $\mathrm{Ni}$ concentration fluctuates around equilibrium after 0.4 seconds until the end of our simulation lasting $1 \mathrm{~s}$.

In order to more accurately estimate the timescale required to obtain the equilibrium profile, we performed five individual KMC simulations with the same equilibrium rate $(40 / \mu \mathrm{s})$, extending the total time to 2.5 second corresponding to about 400 million transitions. Figure 3 shows the averaged Ni composition obtained from these KMC simulations in the bottom three layers (referred as layer 1,2 and 3) and the top three layers (referred as layer 12,11, and 10) with the equilibrium composition profiles obtained by MC together for comparison. From Figure 3a, we can see that layer 1 reaches the equilibrium profile in less than 0.1 second and even approaches zero Ni concentration at $\sim 1.5$ second. Layer 2 exhibits more dramatic changes. The composition started from $30 \% \mathrm{Ni}$, dropped to the equilibrium composition of $10 \mathrm{at} \% \sim 1.75$ second, and remained near the equilibrium until the end of the simulation. While the MC simulation left $16 \% \mathrm{Ni}$ in layer 3, our KMC model shows more Ni segregations leaving $10 \% \mathrm{Ni}$ in the layer. This might explain the higher Ni composition profiles of the top two sublayers (layer 11 and 10) in Figure 3b. While layer 12 shows similar behavior as layer 1, layer 11 did not reach equilibrium until 2.5 second, and layer 10 did reach the equilibrium at $\sim 0.6 \mathrm{~s}$ before the composition increased again. The overall profile, nonetheless, clearly shows an increase in the number of $\mathrm{Ni}$ atoms in bulk layers, indicating thermodynamic tendency for this dopant to remain in the bulk rather than on the surface. 


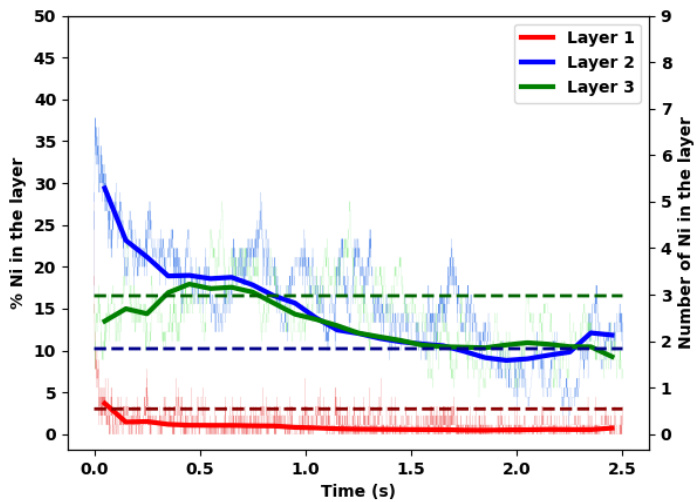

(a)

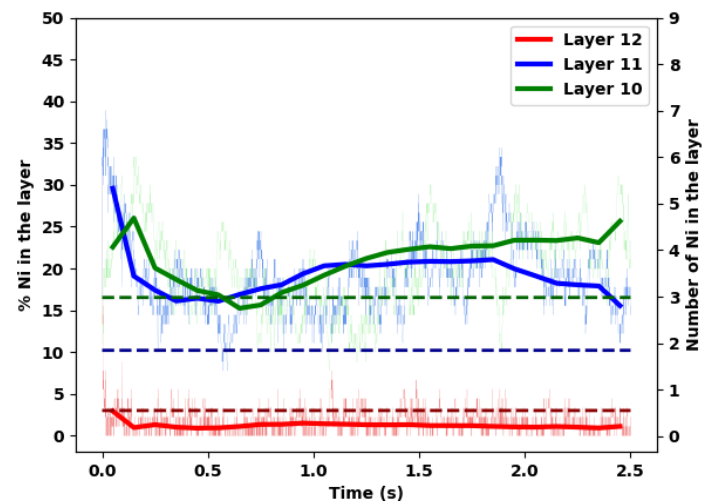

(b)

Figure 3: Ni composition averaged over five KMC simulations for the (a) bottom three layers and (b) top three layers over 2.5 second with an equilibrium rate of $40 / \mu \mathrm{s}$. The bold line represents the 0.5 -second-average calculated from the lighter single-frame datapoints, and the dashed line corresponds to the average concentration of $\mathrm{Ni}$ by MC calculation with EAM potential at each layer when the system reaches equilibrium. Composition is normalized as in Figure 1.

Apart from surface effects, the local chemical environment around a vacancy is expected to determine the system energetics and the rate of Ni composition change. To investigate this, we tracked the number of $\mathrm{Ni}$ atoms within a $5 \AA$ radius of the vacancy. The number of Ni atoms in the local environment ranges from 0 to 12, with three distinct "bins" formed for low, mixed, and high Ni-content environments. The spectrum of barriers calculated during AKMC for all vacancy migration events within this trajectory is presented as a histogram in Figure 4a, which shows the lowest transition state energies for vacancy migration in Ni-rich regions of the alloy. Additionally, because the system has lower $\mathrm{Ni}$ content than $\mathrm{Cu}$, the integrated peak area is smaller for transitions into/within Ni-rich regions than for those with mixed and $\mathrm{Cu}$-rich compositions. Vacancy migration energies are shifted closer to $0.4 \mathrm{eV}$ in the Ni-rich regions than 
for migration in Cu-rich regions according to Figure 4a. We can deduce that vacancy migration is favored in the Ni-rich regions, contributing to the lower dwell time near Ni as the vacancy more rapidly diffuses towards and away from this dopant. Vacancies must slowly explore the $\mathrm{Cu}$-rich regions of the host lattice before returning to possibly segregate the $\mathrm{Ni}$ atoms away from the surface. The order of integrated peak areas in Figure 4a also supports this conclusion.

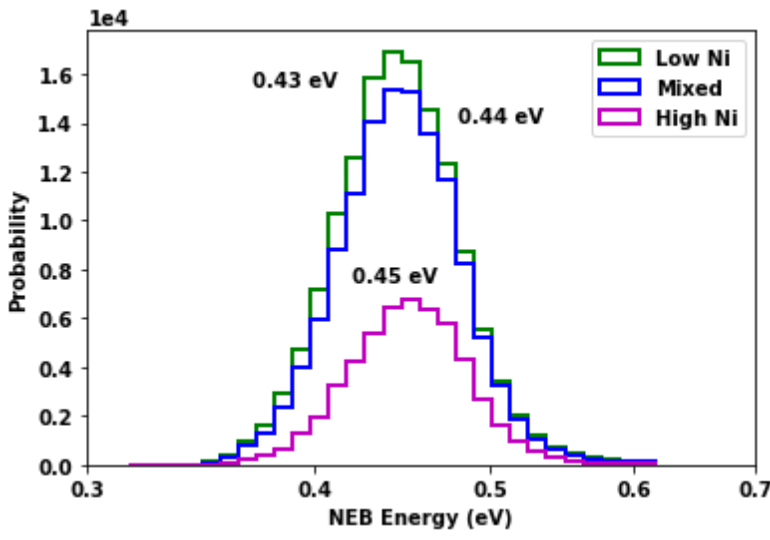

(a)

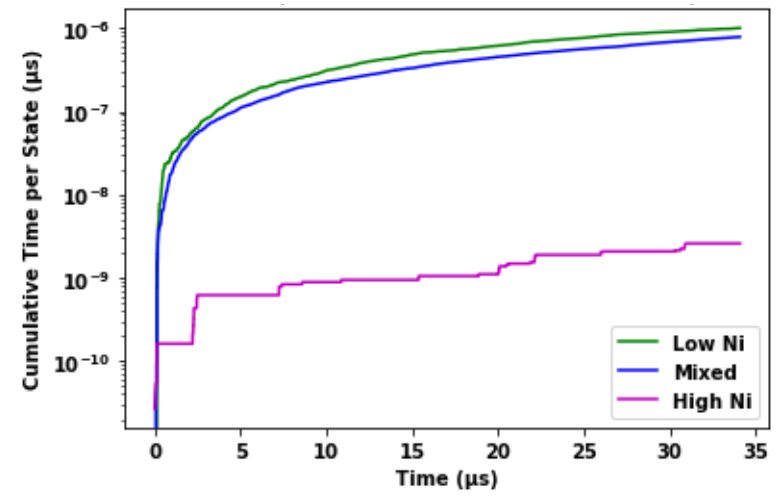

(c)

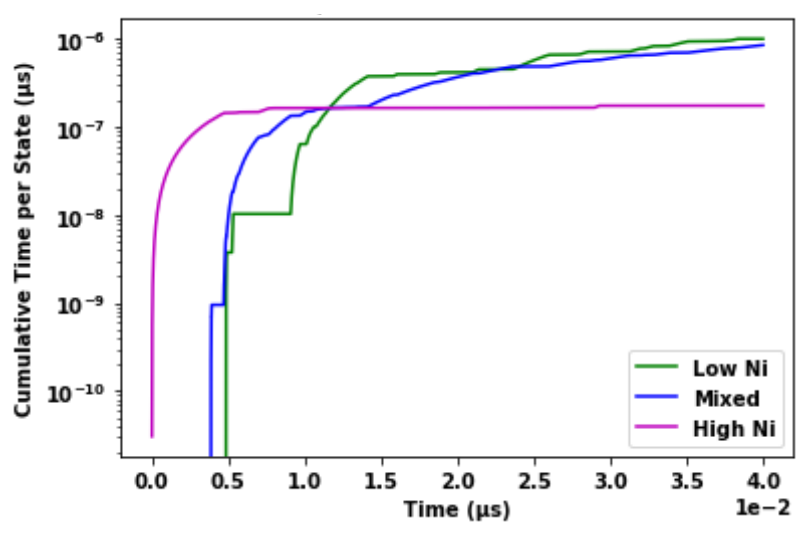

(b)

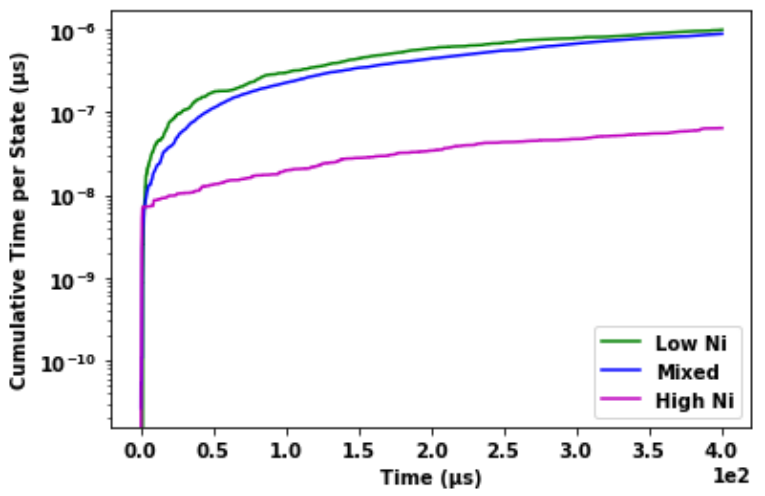

(d)

Figure 4: (a) Histogram of vacancy migration energy barriers obtained from a $319 \mu$ s AKMC simulation. (b-d) Total residence time for the vacancy in different chemical environments for (b) MD, (c) ParSplice, and (d) AKMC. Cumulative times are normalized by the prevalence of each composition type in the system rendering them unphysical. 
The overall time spent in low Ni, mixed, and high Ni content environments is also shown with respect to time in Figure 4b-d using a cumulative measure of residence time for MD, ParSplice, and AKMC and re-scaling this measure by the prevalence of each environment in the system. The number of $\mathrm{Ni}$ atoms in the vicinity of the vacancy modulates these timescales substantially but in similar fashion for all methods. Further, the logarithmic trendlines and their orders of magnitude agree across overlapping timescales for MD/ParSplice (ns) and ParSplice/AKMC ( $\mu$ s): this supports the hierarchy of methods utilized herein as a theoretical foundation to connect correlated observations evolving across many timescales for the same process. The cumulative dwell time and thermodynamic trends match for all of the simulation methods in the order of integrated peak areas (Figure 4a) and residence times (Figure 4b-d): $\mathrm{t}_{\mathrm{Cu}-\text { rich }}^{\text {total }}>\mathrm{t}_{\text {mixed }}^{\text {total }}>\mathrm{t}_{\mathrm{Ni} \text {-rich }}^{\text {total }}$. Though this agreement does not hold at very early (ps) simulation times with low cumulative sums, particularly for MD which is noisier as compared to accelerated methods, the trend becomes evident in the ergodic limit. The residence times of vacancy chemical environment presented in Figure $4 b-d$ confirm that the local composition is an effective determinant of where a vacancy spends most of its time during annealing and segregation. Specifically, the position of $\mathrm{Ni}$ in the lattice is a minor determinant of the dynamics, since the dopant slightly biases the vacancy's random walk by $\sim 0.1 \mathrm{eV}$. This effect has also been documented in Ni-Fe surfaces annealed at $1100 \mathrm{~K}$ using $\mathrm{KMC}$, where the local composition and the identity of the atoms exchanging during segregation (solute vs. solvent) significantly influenced the vacancy migration energy and the measured tracer diffusion coefficient[15]. 


\section{Conclusions}

In summary, we have applied three accelerated dynamics methods to examine the rate of segregation in $\mathrm{CuNi}$ alloy from nanosecond to second timescales, reaching 1 second of simulation time and the equilibrium composition with KMC. This composition profile shows no $\mathrm{Ni}$ on the top surface monolayer, with less than $15 \mathrm{at} \% \mathrm{Ni}$ in the $2^{\text {nd }}$ layer compared to $22 \mathrm{at} \% \mathrm{Ni}$ in the bulk on a per layer basis, in agreement with MC predictions. Though most of our accelerated methods were used to simulate up to the $\mu$ s timescale, only modified KMC dynamics could reach the equilibrium profile obtained from MC and previous experimental observations. Our model estimates that the timescale for segregation in the top layer to reach equilibrium is on the order of $0.1 \mathrm{~ms}$, while equilibrium segregation does not penetrate to the $3^{\text {rd }}$ layer until timescales on the order of $100 \mathrm{~ms}$. The equilibrium timescales for surface segregation of any FCC bimetal can be determined with a combination of AKMC and KMC. However, KMC-based methods require assumptions regarding transition state theory, and they do not resolve fast atomic exchange processes as well as ParSplice and MD. Our study shows that a model for the relationship between solvent distribution, activation energy, and large-scale phenomena like segregation may be developed from further simulations of bimetallics on experimental timescales.

\section{Declaration of Interests}

The authors declare that they have no known competing financial interests or personal relationships that could have appeared or considered to influence the work reported in this paper.

\section{Acknowledgments}

This work was funded from NSF (DMR-1809085, CHE-210231, and CMMI-1905647) as well as resources and collaboration from the University of Pittsburgh's Computational Resource Cluster 
(CRC), the Texas Advanced Computing Center, and the Theoretical Division T-1 of Los Alamos National Laboratory.

\section{References}

1. Bardi, U., The atomic structure of alloy surfaces and surface alloys. Reports on Progress in Physics, 1994. 57(10): p. 939-987.

2. Brongersma, H.H., M.J. Sparnaay, and T.M. Buck, Surface segregation in Cu-Ni and CuPt alloys; A comparison of low-energy ion-scattering results with theory. Surface Science, 1978. 71(3): p. 657-678.

3. Erdélyi, Z., et al., Investigation of the interplay of nickel dissolution and copper segregation in $\mathrm{Ni} / \mathrm{Cu}(111)$ system. Surface Science, 2002. 496(1): p. 129-140.

4. Landa, A., et al., Development of Finnis-Sinclair type potentials for $\mathrm{Pb}, \mathrm{Pb}-\mathrm{Bi}$, and $\mathrm{Pb}-$ Ni systems: application to surface segregation. Acta Materialia, 1998. 46(9): p. 30273032.

5. Jiang, C. and B. Gleeson, Surface segregation of Pt in $\gamma^{\prime}-N i 3 A l$ : A first-principles study. Acta Materialia, 2007. 55(5): p. 1641-1647.

6. Zhevnenko, S.N., et al., Surface and segregation energies of Ag based alloys with Ni, Co and Fe: Direct experimental measurement and DFT study. Acta Materialia, 2021. 205: p. 116565.

7. Voter, A.F., F. Montalenti, and T.C. Germann, Extending the Time Scale in Atomistic Simulation of Materials. Annual Review of Materials Research, 2002. 32(1): p. 321-346. 
8. Miron, R.A. and K.A. Fichthorn, Multiple-Time Scale Accelerated Molecular Dynamics: Addressing the Small-Barrier Problem. Physical Review Letters, 2004. 93(12): p. 128301.

9. Montalenti, F., M.R. Sørensen, and A.F. Voter, Closing the Gap between Experiment and Theory: Crystal Growth by Temperature Accelerated Dynamics. Physical Review Letters, 2001. 87(12): p. 126101.

10. Zamora, R.J., et al., The Modern Temperature-Accelerated Dynamics Approach. Annual Review of Chemical and Biomolecular Engineering, 2016. 7(1): p. 87-110.

11. Perez, D., et al., Long-Time Dynamics through Parallel Trajectory Splicing. Journal of Chemical Theory and Computation, 2016. 12(1): p. 18-28.

12. Henkelman, G. and H. Jónsson, Long time scale kinetic Monte Carlo simulations without lattice approximation and predefined event table. The Journal of Chemical Physics, 2001. 115(21): p. 9657-9666.

13. Lavrentiev, M.Y., et al., Atomistic simulations of surface diffusion and segregation in ceramics. Computational Materials Science, 2006. 36(1): p. 54-59.

14. Li, L., et al., Adaptive kinetic Monte Carlo simulations of surface segregation in PdAu nanoparticles. Nanoscale, 2019. 11(21): p. 10524-10535.

15. Ferasat, K., et al., Accelerated kinetic Monte Carlo: A case study; vacancy and dumbbell interstitial diffusion traps in concentrated solid solution alloys. The Journal of Chemical Physics, 2020. 153(7): p. 074109.

16. Helander, T. and J. Ågren, A phenomenological treatment of diffusion in $\mathrm{Al}-\mathrm{Fe}$ and $\mathrm{Al}-$ Ni alloys having B2-b.c.c. ordered structure. Acta Materialia, 1999. 47(4): p. 1141-1152. 
17. Osetsky, Y.N., L.K. Béland, and R.E. Stoller, Specific features of defect and mass transport in concentrated fcc alloys. Acta Materialia, 2016. 115: p. 364-371.

18. Hennes, M., et al., Equilibrium segregation patterns and alloying in Cu/Ni nanoparticles: Experiments versus modeling. Physical Review B, 2015. 91(24): p. 245401.

19. Webber, P.R., M.A. Morris, and Z.G. Zhang, Crystal-face specificity in surface segregation of CuNi alloys. Journal of Physics F: Metal Physics, 1986. 16(4): p. 413-419.

20. Jin, T., et al., Surface Characterization and Corrosion Behavior of 90/10 Copper-Nickel Alloy in Marine Environment. Materials, 2019. 12(11).

21. Tuck, C.D.S., C.A. Powell, and J. Nuttall, Corrosion of Copper and Its Alloys, in Reference Module in Materials Science and Materials Engineering. 2016, Elsevier.

22. Zegkinoglou, I., et al., Surface Segregation in CuNi Nanoparticle Catalysts During CO2 Hydrogenation: The Role of $\mathrm{CO}$ in the Reactant Mixture. The Journal of Physical Chemistry. C, Nanomaterials and Interfaces, 2019. 123(13): p. 8421-8428.

23. Tan, Q., Z. Shi, and D. Wu, CO2 Hydrogenation to Methanol over a Highly Active CuNi/CeO2-Nanotube Catalyst. Industrial \& Engineering Chemistry Research, 2018. 57(31): p. 10148-10158.

24. Austin, N., B. Butina, and G. Mpourmpakis, CO2 activation on bimetallic CuNi nanoparticles. Progress in Natural Science: Materials International, 2016. 26(5): p. 487492.

25. Lortie, M., et al., Synthesis of $\mathrm{CuNi} / \mathrm{C}$ and $\mathrm{CuNi} /$-Al2O3 Catalysts for the Reverse Water Gas Shift Reaction. International Journal of Chemical Engineering, 2015.

26. Good, B., G. Bozzolo, and J. Ferrante, Surface segregation in Cu-Ni alloys. Physical Review B, 1993. 48(24): p. 18284-18287. 
27. Foiles, S.M., Calculation of the surface segregation of $\mathrm{Ni}$-Cu alloys with the use of the embedded-atom method. Physical Review B, 1985. 32(12): p. 7685-7693.

28. Tréglia, G., B. Legrand, and P. Maugain, Surface segregation in CuNi and AgNi alloys formulated as an area-preserving map. Surface Science, 1990. 225(3): p. 319-330.

29. Panizon, E., et al., Study of structures and thermodynamics of CuNi nanoalloys using a new DFT-fitted atomistic potential. Physical Chemistry Chemical Physics, 2015. 17(42): p. $28068-28075$.

30. Xiang, M., et al., Shock-induced plasticity in semi-coherent $\{111\} \mathrm{Cu}$-Ni multilayers. International Journal of Plasticity, 2018. 103: p. 23-38.

31. Perez, D., B.P. Uberuaga, and A.F. Voter, The parallel replica dynamics method Coming of age. Computational Materials Science, 2015. 100: p. 90-103.

32. Voter, A.F., Parallel replica method for dynamics of infrequent events. Physical Review B, 1998. 57(22): p. 13985-13988.

33. Perez, D., R. Huang, and A.F. Voter, Long-time molecular dynamics simulations on massively parallel platforms: A comparison of parallel replica dynamics and parallel trajectory splicing. Journal of Materials Research, 2018. 33(7): p. 813-822.

34. Le Bris, C., et al., A mathematical formalization of the parallel replica dynamics. Monte Carlo Methods and Applications, 2012. 18(2): p. 119-146.

35. Xu, L. and G. Henkelman, Adaptive kinetic Monte Carlo for first-principles accelerated dynamics. The Journal of Chemical Physics, 2008. 129(11): p. 114104.

36. Henkelman, G. and H. Jónsson, A dimer method for finding saddle points on high dimensional potential surfaces using only first derivatives. The Journal of Chemical Physics, 1999. 111(15): p. 7010-7022. 
37. Kästner, J. and P. Sherwood, Superlinearly converging dimer method for transition state search. The Journal of Chemical Physics, 2008. 128(1): p. 014106.

38. Heyden, A., A.T. Bell, and F.J. Keil, Efficient methods for finding transition states in chemical reactions: Comparison of improved dimer method and partitioned rational function optimization method. The Journal of Chemical Physics, 2005. 123(22): p. 224101.

39. Fischer, F., G. Schmitz, and S.M. Eich, A systematic study of grain boundary segregation and grain boundary formation energy using a new copper-nickel embedded-atom potential. Acta Materialia, 2019. 176: p. 220-231.

40. Kresse, G. and J. Furthmüller, Efficient iterative schemes for ab initio total-energy calculations using a plane-wave basis set. Physical Review B, 1996. 54(16): p. 1116911186.

41. Kresse, G. and J. Furthmüller, Efficiency of ab-initio total energy calculations for metals and semiconductors using a plane-wave basis set. Computational Materials Science, 1996. 6(1): p. 15-50.

42. Kresse, G. and D. Joubert, From ultrasoft pseudopotentials to the projector augmentedwave method. Physical Review B, 1999. 59(3): p. 1758-1775.

43. Perdew, J.P., K. Burke, and M. Ernzerhof, Generalized Gradient Approximation Made Simple. Physical Review Letters, 1996. 77(18): p. 3865-3868.

44. Blöchl, P.E., Projector augmented-wave method. Physical Review B, 1994. 50(24): p. 17953-17979.

45. Ruban, A.V., H.L. Skriver, and J.K. Nørskov, Surface segregation energies in transitionmetal alloys. Physical Review B, 1999. 59(24): p. 15990-16000. 
46. Beichl, I. and F. Sullivan, The Metropolis Algorithm. Computing in Science and Engineering, 2000. 2(1): p. 65-69.

47. Sakurai, T., et al., New result in surface segregation of Ni-Cu binary alloys. Physical Review Letters, 1985. 55(5): p. 514-517.

48. Plimpton, S., Fast Parallel Algorithms for Short-Range Molecular Dynamics. Journal of Computational Physics, 1995. 117(1): p. 1-19.

49. Sheppard, D., R. Terrell, and G. Henkelman, Optimization methods for finding minimum energy paths. The Journal of Chemical Physics, 2008. 128(13): p. 134106.

50. Henkelman, G. and H. Jónsson. EON: Long timescale dynamics. 2021 July 23, 2021]; Available from: https://theory.cm.utexas.edu/eon/.

51. Lam, N.Q., L. Dagens, and N.V. Doan, Calculations of the properties of self-interstitials and vacancies in the face-centred cubic metals $\mathrm{Cu}, \mathrm{Ag}$ and $\mathrm{Au}$. Journal of Physics F: Metal Physics, 1983. 13(12): p. 2503-2516.

52. Novotny, M.A., Monte Carlo Algorithms with Absorbing Markov Chains: Fast Local Algorithms for Slow Dynamics. Physical Review Letters, 1995. 74(1): p. 1-5.

53. Donnelly, R.G. and T.S. King, Surface composition and surface cluster size distribution of Cu-Ni alloys via a monte carlo method. Surface Science, 1978. 74(1): p. 89-108.

54. Eymery, J. and J.C. Joud, Surface segregation in binary Cu-Ni and Pt-Ni alloys using Monte Carlo simulation. Surface Science, 1990. 231(3): p. 419-426.

55. Shumway, R.H. and D.S. Stoffer, Time Series Analysis and Its Applications (Springer Texts in Statistics). 2005: Springer-Verlag.

56. Xiao, P. and G. Henkelman, Kinetic Monte Carlo Study of Li Intercalation in LiFePO4. ACS Nano, 2018. 12(1): p. 844-851. 


\section{Supplementary Information}

\section{Atomistic mechanisms of binary alloy surface segregation from nanoseconds to seconds using accelerated dynamics}

Richard B. Garza ${ }^{1,2}$, Jiyoung Lee ${ }^{3,4}$, Mai H. Nguyen ${ }^{3}$, Andrew Garmon ${ }^{5,6}$, Danny Perez ${ }^{5}$, Meng $\mathrm{Li}^{2}$, Judith C. Yang ${ }^{2}$, Graeme Henkelman ${ }^{3,4}$, Wissam A. Saidi ${ }^{*}$

${ }^{1}$ Department of Mechanical Engineering and Materials Science, University of Pittsburgh, Pittsburgh, PA

${ }^{2}$ Department of Chemical and Petroleum Engineering, University of Pittsburgh, Pittsburgh, PA

${ }^{3}$ Department of Chemistry, University of Texas at Austin, Austin, TX

${ }^{4}$ Oden Institute for Computational Engineering \& Sciences, University of Texas at Austin, Austin, TX

${ }^{5}$ Theoretical Division T-1, Los Alamos National Laboratory, Los Alamos, NM

${ }^{6}$ Department of Physics \& Astronomy, Clemson University, Clemson SC

*Corresponding author. E-mail: alsaidi@pitt.edu

\section{Appendix A: EAM Validation Using DFT}

The surface energies of doped $\mathrm{Cu}$ surfaces up to $2.7 \mathrm{at} \% \mathrm{Ni}$ were computed using DFT and the EAM potential of focus in this work: this was done not only to validate the forcefield's 
application in the study of surface segregation, but also to examine the process with ab initio accuracy[39]. We used the Vienna Ab Initio Package (VASP) [40-42, 44] in conjunction with Perdew-Burke-Ernzerhof (PBE) [43] exchange-correlation functional and an energy cutoff of $500 \mathrm{eV}$ to expand the wavefunction. We used a $3 \times 3 \times 1 \mathrm{k}$-point mesh generated with a Monkhorst-Pack routine to sample the Brillouin zone for $<100>,<110>$, and $<111>$ slab models composed of $\sim 100$ atoms. All calculations are spin polarized. We used a slab approach to model the surfaces and included $10 \AA$ of vacuum between exposed surfaces to mitigate spurious interactions in the non-periodic direction. One of the host lattice atoms in the system $(\mathrm{Cu})$ were replaced with $\mathrm{Ni}$ at both surface ( $1^{\text {st }}$ layer) and subsurface $\left(2^{\text {nd }}\right.$ layer) positions. The surface energy of a pure $\mathrm{Cu}$ slab model with (hkl) orientation including $\mathrm{N}_{\text {atoms }}$ is calculated relative to the bulk energy per atom $\mathrm{E}_{\mathrm{Cu}}$ and the total exposed area $2 \mathrm{~A}$ :

$$
\mathrm{E}_{\mathrm{hkl}}^{\text {surf }}=\frac{\mathrm{E}_{\mathrm{f}}^{\text {pure }}-\mathrm{N}_{\text {atoms }} \mathrm{E}_{\mathrm{Cu}}^{\text {bulk }}}{2 \mathrm{~A}} .
$$

The equation for surface energy of models containing one dopant atom is very similar to the above, and requires the bulk energy per atom of the dopant ( $\mathrm{Ni}$ in this study):

$$
\mathrm{E}_{\mathrm{hkl}}^{\text {surf }}=\frac{\mathrm{E}_{\mathrm{f}}^{\text {doped }}-\left(\mathrm{N}_{\text {atoms }}-1\right) \mathrm{E}_{\mathrm{Cu}}^{\mathrm{bulk}}-\mathrm{E}_{\mathrm{Ni}}^{\text {bulk }}}{2 \mathrm{~A}}
$$

The pure $\mathrm{Cu}$ surface energies for orientations with low Miller index $\left(\mathrm{h}^{2}+\mathrm{k}^{2}+\mathrm{l}^{2}<3\right)$ are shown in Table A1, which were calculated with DFT and EAM. As is known from previous calculations and measurements of surface energy for pure $\mathrm{Cu},<111>$ surfaces are most thermodynamically stable, followed by $<100>$ and $<110>$ surfaces. The validation with pure $\mathrm{Cu}$ surfaces finds $2.6 \%$ average relative error between the EAM potential and first-principles. We then replaced one atom in these structures with $\mathrm{Ni}$ to study the effect of doping at different surface depths. 


\begin{tabular}{|c|c|c|}
\hline & DFT $\left(\mathbf{J} / \mathbf{m}^{2}\right)$ & $\operatorname{EAM}\left(J / \mathbf{m}^{2}\right)$ \\
\hline $\mathrm{Cu}(\mathbf{1 0 0})$ & 1.412 & 1.357 \\
\hline $\mathrm{Cu}(\mathbf{1 1 0})$ & 1.477 & 1.488 \\
\hline $\mathrm{Cu}(111)$ & 1.208 & 1.246 \\
\hline
\end{tabular}

Table A1: Surface energies of pure $\mathrm{Cu}$ from DFT and the EAM potential of Fischer et al Oriented slabs along (100), (110), and (111) planes exhibit segregation trends as a function of crystallographic direction in the FCC system for $\mathrm{Ni}$, evident in Table A2. EAM calculations of $\mathrm{Cu}$ surface energy with an included $\mathrm{Ni}$ dopant at various locations have only 4.7\% average relative error compared to DFT results. Additionally, the difference in minimized energies between structures with $\mathrm{Ni}$ on the exposed surface or beneath it (subsurface) is 0.38 $0.39 \mathrm{eV}$ for $<100>$ and $<111>\mathrm{Cu}$ surfaces, yet this gap is only $0.17 \mathrm{eV}$ for doped $<111>\mathrm{Cu}$ surface. The same trend was found to cause the equilibrium segregation profiles from previous $\mathrm{MC}$ studies of CuNi surface separation, agreeing with the calculated energetics in our own validation step[18, 26, 27, 53, 54]. Overall, the trend indicates that $\mathrm{Ni}$ will segregate towards subsurface sites to relax the local surface tension.

\begin{tabular}{ccccccc} 
& \multicolumn{2}{c}{ CuNi(100) } & \multicolumn{2}{c}{ CuNi(110) } & \multicolumn{2}{c}{ CuNi(111) } \\
\hline \multirow{2}{*}{ Layer \# } & DFT & EAM & DFT & EAM & DFT & EAM \\
& $\left(\mathbf{J} / \mathbf{m}^{2}\right)$ & $\left(\mathbf{J} / \mathbf{m}^{2}\right)$ & $\left(\mathbf{J} / \mathbf{m}^{2}\right)$ & $\left(\mathbf{J} / \mathbf{m}^{2}\right)$ & $\left(\mathbf{J} / \mathbf{m}^{2}\right)$ & $\left(\mathbf{J} / \mathbf{m}^{2}\right)$ \\
\hline 1st Layer & 1.387 & 1.371 & 1.457 & 1.512 & 1.165 & 1.264 \\
\hline 2nd Layer & 1.360 & 1.365 & 1.430 & 1.505 & 1.150 & 1.256 \\
\hline 3rd Layer & 1.364 & 1.362 & 1.429 & 1.501 & 1.152 & 1.257 \\
\hline
\end{tabular}


Table A2: Surface energies of $\mathrm{Cu}$ with 1x Ni dopant atom included on the surface, subsurface, or interior $\left(1^{\text {st }}, 2^{\text {nd }}\right.$, or $3^{\text {rd }}$ layer $)$ to illustrate segregation trends from DFT and EAM

The monovacancy formation energies for pure $\mathrm{Cu}$ and pure $\mathrm{Ni}$ are $1.29 \mathrm{eV}$ and $1.57 \mathrm{eV}$, respectively.

\section{Appendix B: Composition Profile Estimation MC Data}

Each step of the simulated annealing to equilibrium is dependent on the previous configuration (serial correlation), so the observed samples for calculating the estimated mean composition have a biased standard error that is overestimated by a naïve error calculation. To correct this, autocorrelation of subsequent frames in the MC process was estimated by assuming samples are generated by a first order autoregressive process (AR(1)):

$$
\mathrm{X}_{\mathrm{t}}=\mathrm{c}+\rho \mathrm{X}_{\mathrm{t}-1}+\varepsilon_{\text {unbiased }}
$$

where $X_{t}$ is the estimated value at time $t$ by the $A R(1)$ model, $c$ is an optional shifting constant (typically zero), and $\varepsilon$ the corrected error we wish to estimate. The process has an analytically known correction to find the unbiased, standard error of the estimated mean from $n$ measurements and depends on $\rho$ to calculate the measurement error for each layer[55]:

$$
\begin{gathered}
\sigma_{\text {unbiased }}=\sigma_{\text {biased }} \sqrt{\frac{1+2 \delta / n}{1-\frac{2 \delta}{n(n-1)}}} \\
\delta=\frac{(n-1) \rho-n \rho^{2}+\rho^{n+1}}{(1-\rho)^{2}}
\end{gathered}
$$

A custom Perl script was used to analyze the output data from MC, finding the composition in each layer and tracking it over all the annealed samples. These measurements were exported to a Python script which estimated values of $\rho$ for the data using the 'statsmodels' 
module, and the above equations estimated accurate errors for plots of the equilibrium composition profiles.

\section{Appendix C: Cluster Expansion Model for Kinetic Monte Carlo (KMC) simulation}

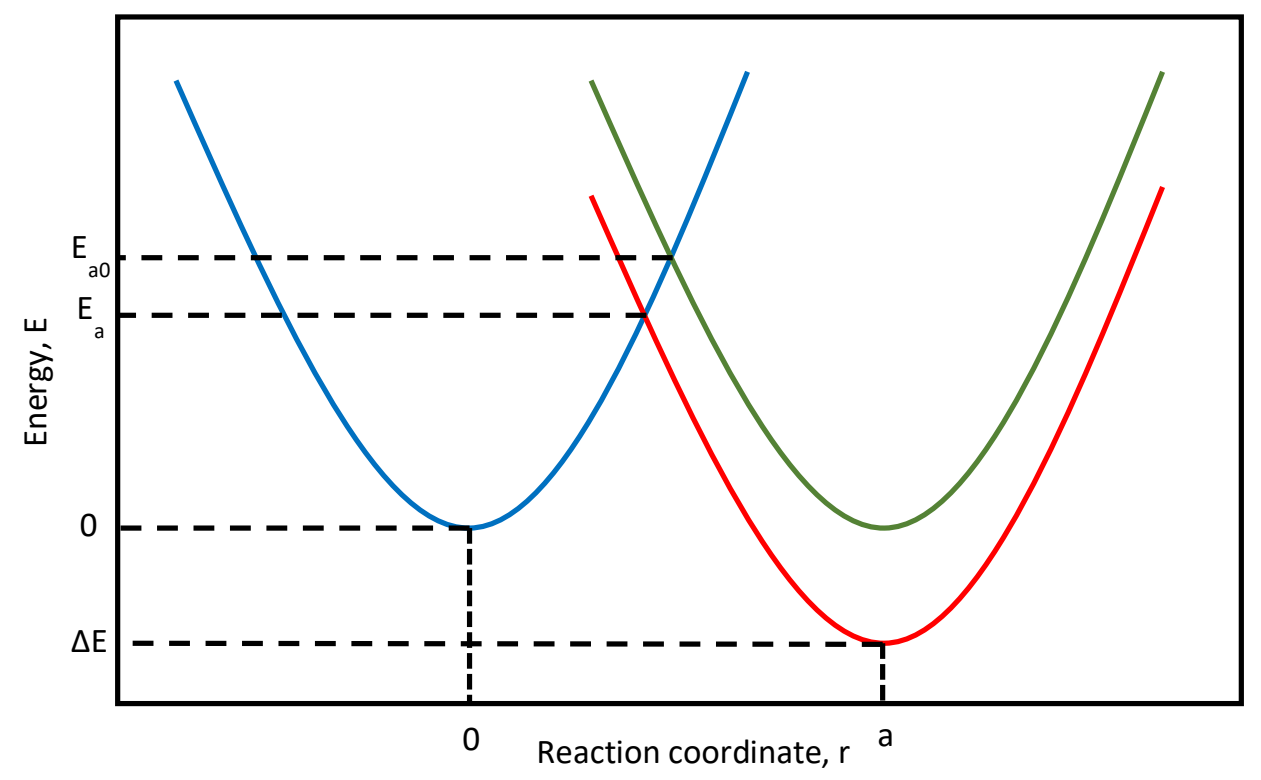

Figure C1: Parabolic construction showing energy diagram of a system. The blue curve is the initial state, the green curve is the final state with the same energy as the blue curve while the red curve is the final state with lower energy than the blue curve (Recreation)[56].

The reaction coordinate between two states is identified and analyzed using high-temperature sampling and climbing image nudged elastic band (CI-NEB) in AKMC. Contrastingly, our KMC model approximates these transition energies using an equation parametrized from barriers already known from AKMC: in Figure C1, the energy of the red curve is lower than the green 
curve due to a change in local environment of the vacancy. As a result, the barrier is lower and the rate can be calculated analytically as [56]

$$
\begin{gathered}
\mathrm{E}_{\mathrm{a}}=\frac{\left(4 \mathrm{E}_{\mathrm{a} 0}+\Delta \mathrm{E}\right)^{2}}{16 \mathrm{E}_{\mathrm{a} 0}} \\
\text { rate }=A \mathrm{e}^{\frac{-\mathrm{E}_{\mathrm{a}}}{\mathrm{kT}}}
\end{gathered}
$$

where $E_{a}$ is the barrier from one state to a lower-energy state (blue and red), $E_{a 0}$ is the barrier between two isoenergetic states (blue and green) also known as the intrinsic barrier, $\Delta \mathrm{E}$ is the energy difference between the initial and the final state, $\mathrm{A}$ is the pre-factor in $\mathrm{s}^{-1}, \mathrm{k}$ is the Boltzmann constant $8.617 \mathrm{eV} \cdot \mathrm{K}^{-1}$, and $\mathrm{T}$ is temperature in $\mathrm{K}$. The intrinsic barrier $\mathrm{E}_{\mathrm{a} 0}$ is obtained from AKMC data in Table C1.

Table C1: Intrinsic barrier of a vacancy migration event taken from AKMC data

\begin{tabular}{ccccc}
\hline $\begin{array}{c}\text { Location } \\
\text { of the }\end{array}$ & Surface & \multicolumn{2}{c}{ Subsurface } \\
vacancy & & & & \\
\hline $\begin{array}{c}\text { Moving } \\
\text { atom }\end{array}$ & $\mathrm{Cu}$ & $\mathrm{Ni}$ & $\mathrm{Cu}$ & $\mathrm{Ni}$ \\
\hline Intrinsic & & & & \\
barrier & 0.453 & 0.487 & 0.635 & 0.681 \\
$\mathbf{E}_{\mathbf{a} 0}(\mathbf{e V})$ & & & & \\
\hline
\end{tabular}

To acquire $\Delta \mathrm{E}$, the energy of each state is needed for the equation

$$
\Delta \mathrm{E}=\mathrm{E}_{\mathrm{v}, \mathrm{i}}-\mathrm{E}_{\mathrm{v}, \mathrm{j}}
$$


where $E_{v, i}$ is the total energy of the configuration with the vacancy at location $i$ (final) and $E_{v, i}$ is the total energy of the configuration with the vacancy at location $\mathrm{j}$ (initial). Vacancy formation energy is calculated by

$$
E_{f}=E_{v}-E_{0}+\mu_{a}
$$

where $E_{f}$ is the vacancy formation energy, $E_{v}$ is the total energy of the configuration with the vacancy, $\mathrm{E}_{0}$ is the total energy of the configuration without the vacancy (initial configuration) and $\mu_{\mathrm{a}}$ is the chemical potential of the atom removed from the initial configuration to make the vacancy.

The vacancy formation energy can then be used to determine $\Delta \mathrm{E}$ :

$$
\Delta E=\left(E_{f, i}+E_{0}-\mu_{a}\right)-\left(E_{f, j}+E_{0}-\mu_{a}\right)=E_{f, i}-E_{f, j}
$$

Vacancy formation energy is predicted by the cluster expansion method, which samples multiple alloy structures uniformly at random vacancies with different local environments. The energies of these structures were calculated and used to parametrize the model shown in Figure C2.

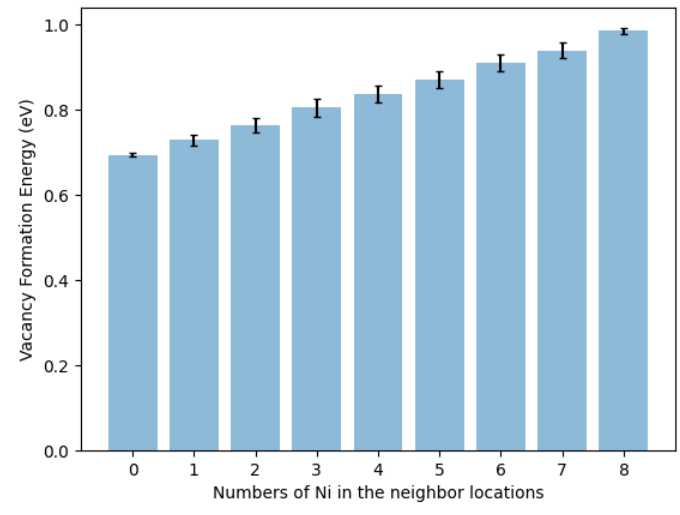

a)

b)

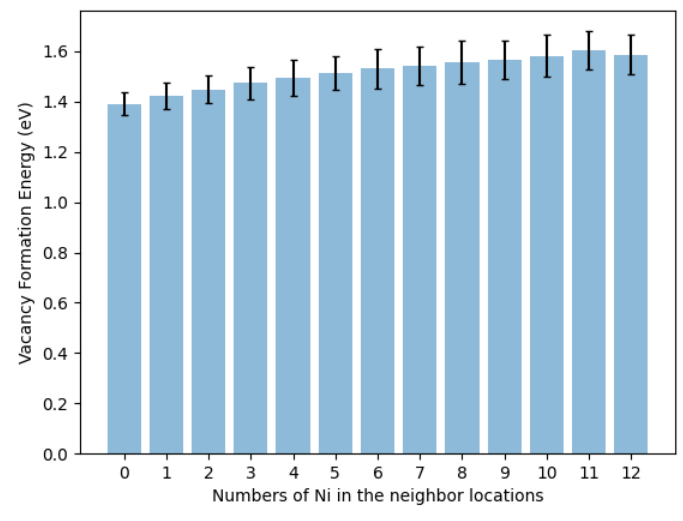



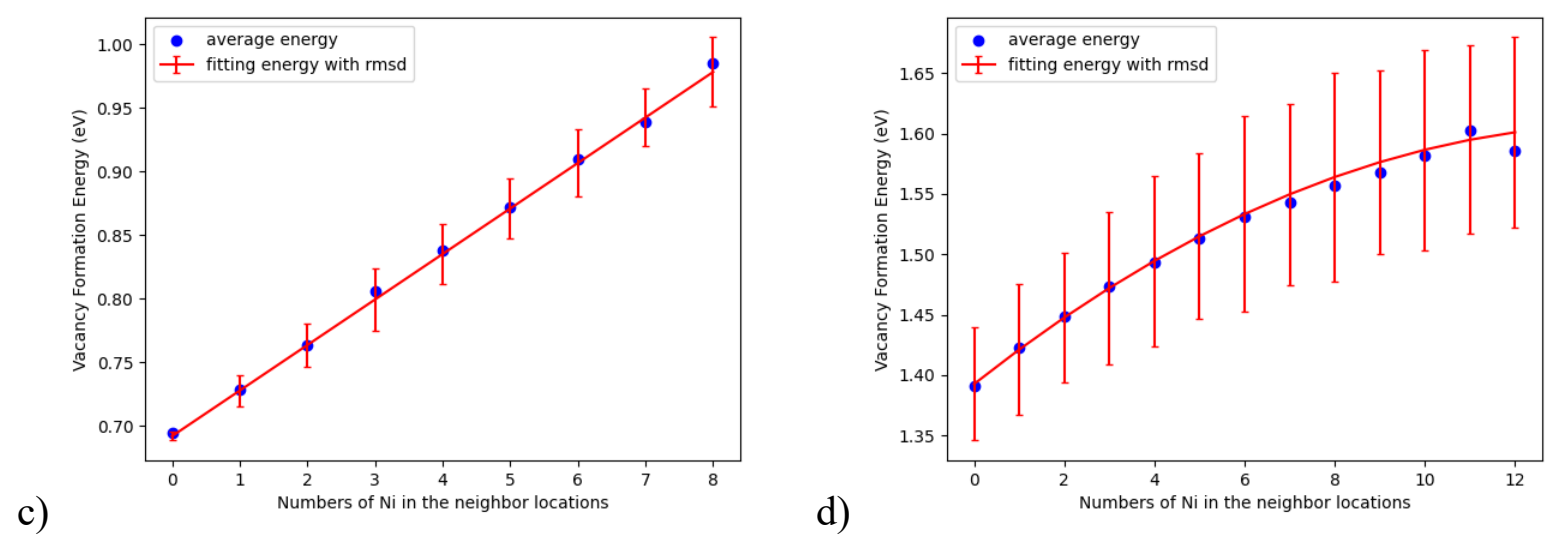

Figure C2: Average and standard deviation values of vacancy formation energy at (a) surface and (b) subsurface. Cluster expansion fitting model for the vacancy formation energy at (c) surface and (d) subsurface.

While the cluster expansion model for KMC works for surface-surface and subsurfacesubsurface diffusion, it fails to illustrate correct surface-subsurface migrations (Figure C2b) since the identity of the migrating atom (counter to the vacancy movement) is not included in this model: Ni requires higher barriers $(\sim 0.95-1.0 \mathrm{eV})$ than $\mathrm{Cu}(\sim 0.8-0.9 \mathrm{eV})$ to move from surface to subsurface according to AKMC data. To fix this significant qualitative limitation of the cluster expansion model, a new definition for the barriers of a vacancy migration from surface to subsurface is proposed based on the AKMC data:

$$
\mathrm{E}_{\mathrm{a}, \mathrm{surf}-\text { sub }}=\Delta \mathrm{E}+\mathrm{E}_{\mathrm{a}, \text { sub-surf }}
$$

The AKMC data shows that the barriers of a vacancy migration from surface to subsurface are very similar to the energy difference of final and initial states ( $\Delta \mathrm{E}$ in the equation above), and values of , $\mathrm{E}_{\mathrm{a}, \text { sub-surf }}$ are very small $(0.049 \mathrm{eV}$ for moving $\mathrm{Cu}$ atom and $0.067 \mathrm{eV}$ for moving $\mathrm{Ni}$ atom) regardless of local environment. Figure $\mathrm{C} 3$ depicts an interpretation to calculate the 
barriers of a vacancy migration from surface to subsurface by adding the difference in energy, $\Delta \mathrm{E}$, between two states to $\mathrm{E}_{\mathrm{a}, \text { sub-surf }}$.

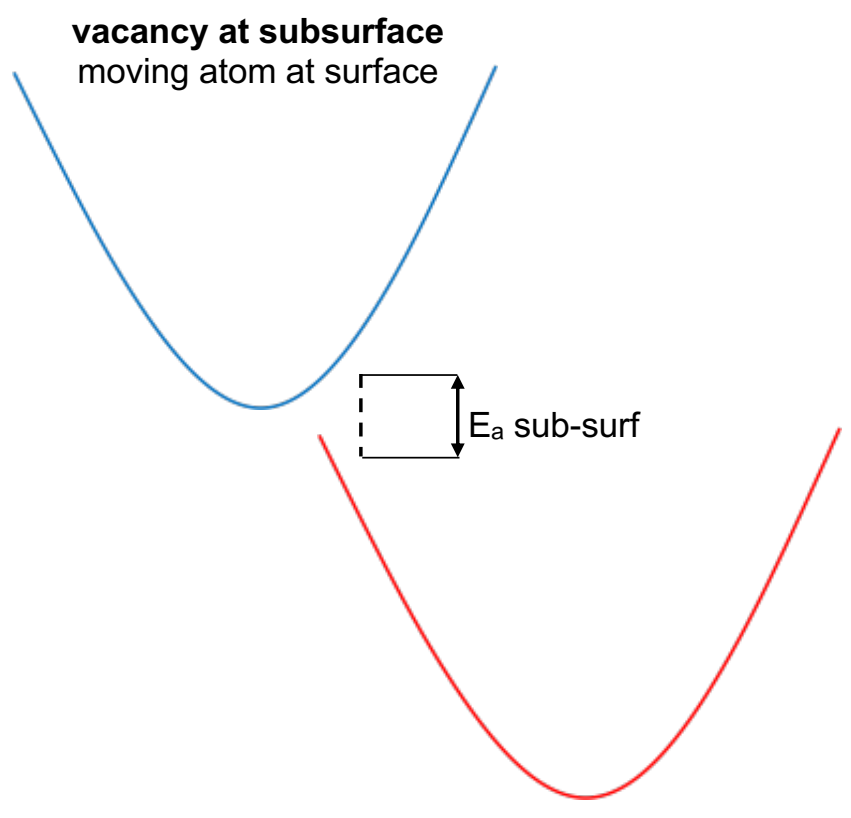

Figure C3. An illustration to determine the barrier of vacancy migration from surface to subsurface using the existed barrier of vacancy migration from subsurface to surface.

By the new definition, the barrier difference between $\mathrm{Ni}$ and $\mathrm{Cu}$ originates from the energy difference of two states $\Delta \mathrm{E}$, and its correction is significant to obtain correct dynamics. To include this information, $\triangle \mathrm{E}$ was calculated from EON, where AKMC runs, then compared with the numerically calculated $\Delta \mathrm{E}$ from cluster expansion. This discrepancy is illustrated in Figure $\mathrm{C} 4[50]$ : the errors are approximately $0.09 \mathrm{eV}$ for $\mathrm{Cu}$ and $0.24 \mathrm{eV}$ for $\mathrm{Ni}$, so these correction values were chosen to improve the cluster expansion model. 

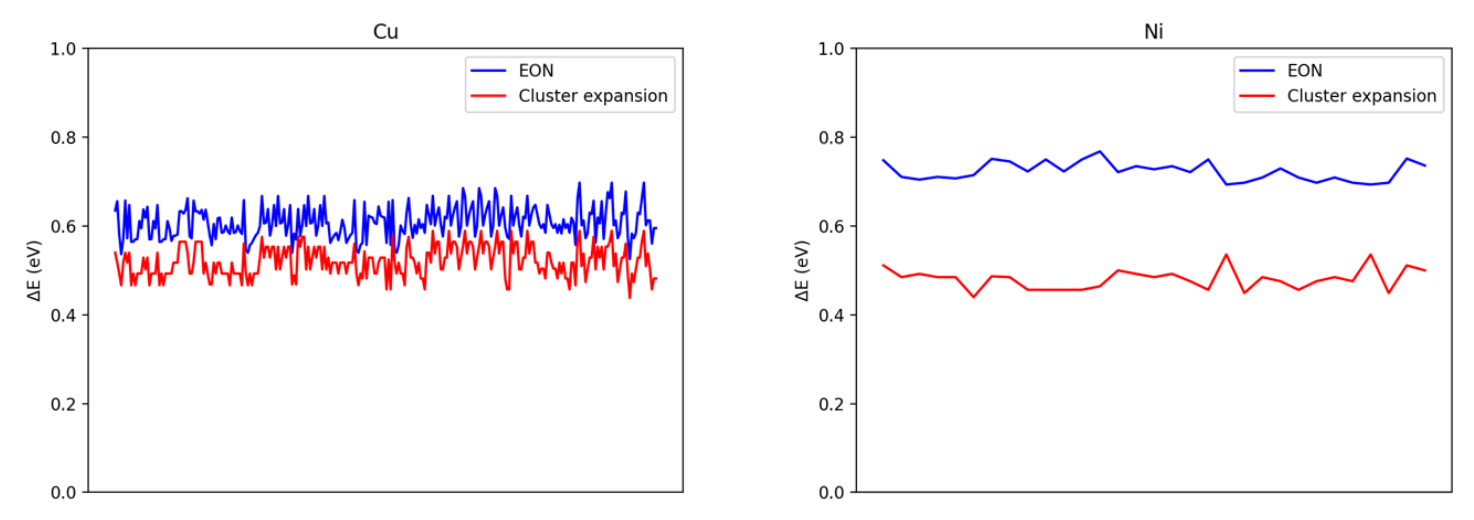

Figure C4: An illustration of discrepancy between $\Delta \mathrm{E}$ from $\mathrm{EON}$ and from cluster expansion

\section{Appendix D: Comparison of AKMC and KMC results}

Figure D1 presents the energy profiles obtained from AKMC and KMC simulations that reach timescale of $300 \mu \mathrm{s}$, realizing 144,597 and 124,650 transition states respectively. Both methods show two Ni segregation events away from the surface at $100 \sim 125 \mu$ s and $170 \sim 200 \mu$ s, which corresponds to the energy drops in Figure. This comparison supports that our KMC model can simulate the similar behaviors that were observed in AKMC.

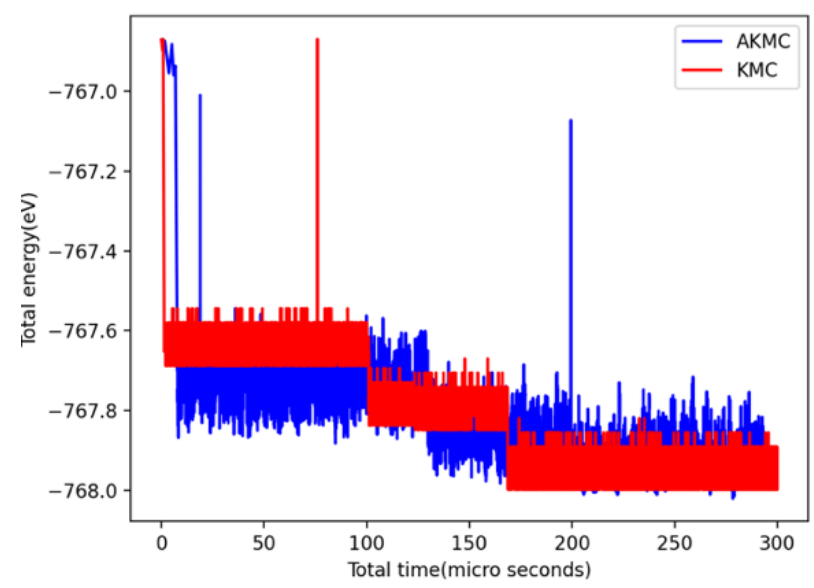

Figure D1: Energetic profiles of AKMC (blue) and KMC (red) simulations with respect to the total time of $300 \mu \mathrm{s}$. 


\section{References:}

[1] F. Fischer, G. Schmitz, S.M. Eich, A systematic study of grain boundary segregation and grain boundary formation energy using a new copper-nickel embedded-atom potential, Acta Materialia 176 (2019) 220-231.

[2] G. Kresse, J. Furthmüller, Efficiency of ab-initio total energy calculations for metals and semiconductors using a plane-wave basis set, Computational Materials Science 6(1) (1996) 1550.

[3] G. Kresse, J. Furthmüller, Efficient iterative schemes for ab initio total-energy calculations using a plane-wave basis set, Physical Review B 54(16) (1996) 11169-11186.

[4] G. Kresse, D. Joubert, From ultrasoft pseudopotentials to the projector augmented-wave method, Physical Review B 59(3) (1999) 1758-1775.

[5] P.E. Blöchl, Projector augmented-wave method, Physical Review B 50(24) (1994) 1795317979.

[6] J.P. Perdew, K. Burke, M. Ernzerhof, Generalized Gradient Approximation Made Simple, Phys. Rev. Lett. 77(18) (1996) 3865-3868.

[7] M. Hennes, J. Buchwald, U. Ross, A. Lotnyk, S.G. Mayr, Equilibrium segregation patterns and alloying in $\mathrm{Cu} / \mathrm{Ni}$ nanoparticles: Experiments versus modeling, Physical Review B 91(24) (2015) 245401.

[8] B. Good, G. Bozzolo, J. Ferrante, Surface segregation in Cu-Ni alloys, Physical Review B 48(24) (1993) 18284-18287.

[9] S.M. Foiles, Calculation of the surface segregation of $\mathrm{Ni}-\mathrm{Cu}$ alloys with the use of the embedded-atom method, Physical Review B 32(12) (1985) 7685-7693. 
[10] R.G. Donnelly, T.S. King, Surface composition and surface cluster size distribution of CuNi alloys via a monte carlo method, Surface Science 74(1) (1978) 89-108.

[11] J. Eymery, J.C. Joud, Surface segregation in binary Cu-Ni and Pt-Ni alloys using Monte Carlo simulation, Surface Science 231(3) (1990) 419-426.

[12] R.H. Shumway, D.S. Stoffer, Time Series Analysis and Its Applications (Springer Texts in Statistics), Springer-Verlag2005.

[13] P. Xiao, G. Henkelman, Kinetic Monte Carlo Study of Li Intercalation in LiFePO4, ACS Nano 12(1) (2018) 844-851.

[14] G. Henkelman, H. Jónsson, EON: Long timescale dynamics. https://theory.cm.utexas.edu/eon/, 2021 (accessed July 23, 2021.). 